\title{
Fiber Bragg Grating Strain Sensors for Marine Engineering
}

\author{
Tingting WANG ${ }^{1}$, Zilin YUAN ${ }^{1 *}$, Yuan $\mathrm{GONG}^{1}, \mathrm{Yu} \mathrm{WU}^{1}$, Yunjiang RAO ${ }^{1}$, Lili WEI $^{1}$, \\ Peng GUO ${ }^{1}$, Junpu $\mathrm{WANG}^{2}$, and $\mathrm{Fu} \mathrm{WAN}^{2}$
}

\author{
${ }^{1}$ Key Laboratory of Optical Fiber Sensing \& Communications (Ministry of Education), University of Electronic Science \\ and Technology of China, Chengdu, 611731, China \\ ${ }^{2}$ Safety Environment Quality Surveillance \& Inspection Research Institute of SPA, Guanghan, 611731, China \\ *Corresponding author: Zilin YUAN_ E-mail: gong.yuan@163.com
}

\begin{abstract}
For the health monitoring of the offshore drilling platform in the salt-fog environment, three nonmetallic materials, i.e., silica, FR-4 epoxy board and sheet molding compound (SMC), with the good anti-corrosion ability were chosen as the packaging materials for the fiber Bragg grating (FBG) strain sensors. By selecting a highly sensitive structure of the fiber Bragg grating strain sensor, the performances of the sensors with three materials were investigated both numerically and experimentally. The strain sensitivities were $3.76 \mathrm{pm} / \mu \varepsilon, 3.02 \mathrm{pm} / \mu \varepsilon$ and $3.03 \mathrm{pm} / \mu \varepsilon$, respectively. The linearity was better than 0.998 . It provides useful information for developing sensors for the marine engineering.
\end{abstract}

Keywords: Offshore drilling platform, fiber Bragg grating, strain sensor, nonmetallic materials

Citation: Tingting WANG, Zilin YUAN, Yuan GONG, Yu WU, Yunjiang RAO, Lili WEI, et al., "Fiber Bragg Grating Strain Sensors for Marine Engineering," Photonic Sensors, DOI: 10.1007/s13320-013-0123-6.

\section{Introduction}

The offshore platform is the key infrastructure for the offshore oil exploration. In the complex and harsh marine environments, the offshore drilling platform will be affected by the wind, waves, sea ice, ocean currents, tides and earthquakes. In addition, the environmental corrosion, adhesion of the sea creatures, passing ships collision, aging of materials, component defects, mechanical damage will also threaten the safety of the drilling platform as a result of the material's fatigue accumulation and resistance attenuation. The damage of the offshore platform structure will not only cause significant economic losses and casualties, but also cause the serious environmental pollution and social problems.
Therefore, it is very important to determine the damage location and degree of injury, as well as to estimate the residual life and carry out the engineering maintenance on the offshore platform with the assist of the effective, reliable and real-time health monitoring.

Fiber Bragg grating (FBG) sensors have many advantages over conventional electric sensors, including the high sensitivity, immunity to electromagnetic interference, and massive multiplexing capability [1]. They are widely used in the aerospace, intelligent transportation, petrochemical, electric power industries, structural health monitoring (SHM) and other military and civilian applications to do real-time online monitoring of the strain, temperature and other

Received: 1 June 2013 / Revised version: 20 June 2013

(C) The Author(s) 2013. This article is published with open access at Springerlink.com 
parameters of engineering structures. FBG sensors are especially fit for the technical requirements of the offshore platform structure monitoring on the good anti-corrosion ability, long-term operation and quasi-distributed sensing $[2,3]$.

The concentration of salt in the ocean atmosphere is much higher than that in the inland areas. In the salt frog environment, the FBG sensors with metal packages are easily to be corrupted and can not work for a long term. Therefore, when selecting the packaging material for the FBG sensor, nonmetallic materials with high corrosion resistance are preferred. Referring to the materials used in marine vessels, oil pipelines and other marine equipments, the commonly used corrosion-resistant and aging-resistant non-metallic materials are polytetrafluoroethylene (PTFE), organic glass and composite materials. PTFE has a large thermal expansion coefficient and is difficult to recover after deformation. Therefore in the marine environment with a large temperature variation or large-strain occasion, the accuracy and durability of sensors packaged with PTFE will be affected. Organic glass has poor temperature tolerance and is easy to get permanent deformation or qualitative change during the packaging process. As a kind of non-magnetic, anti-fatigue, and anti-aging material, the glass fiber reinforced plastic (GFRP) has the good corrosion resistance and high mechanical strength [4]. Therefore, the GFRP is widely used in large-scale engineering structures. Combining the advantages of glass fibers and plastics, this composite material has been extensively investigated and used. In addition, being both physically and chemically stable, silica has the good temperature tolerance, corrosion resistance, small thermal expansion coefficient, highly insulating property, etc. As the silica material has the same characteristics as the fiber itself, it has been widely used in the FBG sensor packaging.

In this paper, we both numerically and experimentally investigate the sensing performance of FBG strain sensors packaged with silica and two kinds of fiber reinforced polymer (FRP) materials, respectively. The packaging structure is similar to the one used in [5]. This paper provides valuable information for the further research on corrosion-resistant and high- performance FBG strain sensors applied in the marine environment.

\section{Sensor structure}

The Bragg wavelength is given by

$$
\lambda_{B}=2 n_{\text {eff }} \Lambda
$$

where $n_{\text {eff }}$ is the effective refractive index of the fiber core, and $\Lambda$ is the period of the index modulation. The wavelength shift, $\Delta \lambda_{B}$, for an applied longitudinal strain is given by

$$
\Delta \lambda_{B}=\lambda_{B}\left(1-p_{e}\right) \varepsilon_{z}
$$

where $p_{e}$ is the photo-elastic coefficient of the fiber, and $\varepsilon_{z}$ is the strain. For the silica single-mode fiber, $p_{e}=0.22$. The wavelength-strain sensitivity of the FBG in silica fiber near $1550 \mathrm{~nm}$ was about $1.2 \mathrm{pm} / \mu \varepsilon[6,7]$.

Figure 1 shows the schematic structure of the FBG strain sensor used in the experiments. The purpose of the area 5 in the structure was to converge the strain of the substrate on the FBG fixed in the center and further to improve the sensitivity of the sensor.

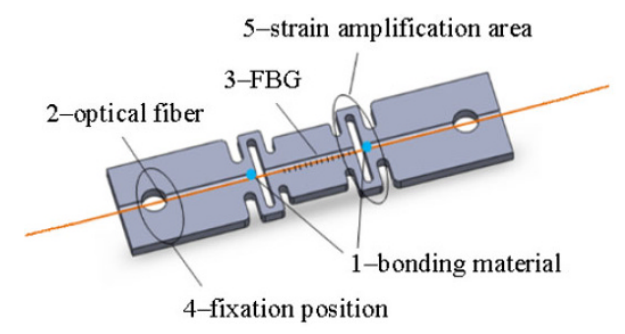

Fig. 1 Schematic structure of the FBG strain sensor.

The strain distribution of the structure under the stress was modeled by the ANSYS finite element software. We set parameters of each strain gauge according to the material properties. Constraints and loads were imposed on the fixed parts of the two ends of the carrier. The strain and displacement distributions were simulated, as shown in Figs. 2 and 3 , respectively. 


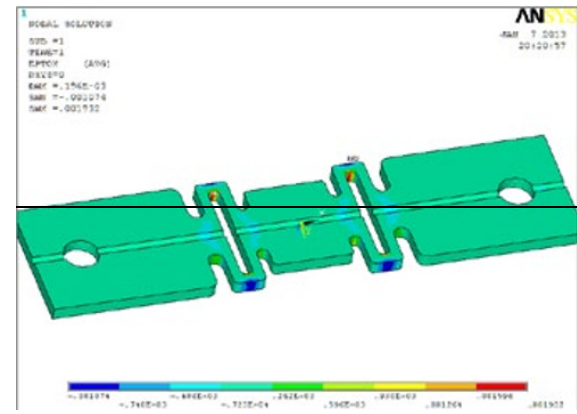

Fig. 2 Strain contour plot of the FBG strain sensor.

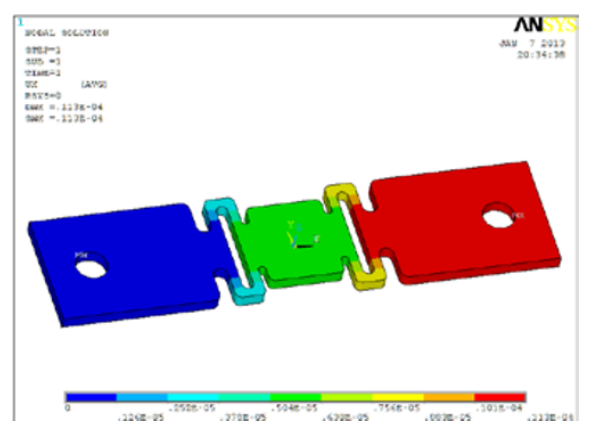

Fig. 3 Displacement contour plot of the FBG strain sensor.

The strain in the structure was mainly concentrated on the area 5 as shown in Fig. 1, which made the entire change in the displacement concentrate on the central part of the substrate where the grating was located. Thus, the strain sensitivity of the packaged FBG sensor was enhanced. There were two holes along the longitudinal axis. During the simulation, one hole was fixed, and the load was applied to the other hole. The three materials used in this experiment were silica, FR-4 GFRP and sheet molding compound (SMC). The Young's modulus, Poisson's ratio and density were set respectively according to the three materials when modeling the structure with the ANSYS software. The simulated and experimental results are given in the next sections.

\section{Simulated results}

The strain between the two holes was calculated as a function of the applied load, as shown in Fig. 4. The strain-load curves are shown for three different materials with the solid line, dashed line and dotted line, respectively. In Fig. 4, the slopes of three curves corresponding to silica, FR-4 and SMC are $21.80 \mu \varepsilon / \mathrm{N}, 105.8 \mu \varepsilon / \mathrm{N}$ and $116.5 \mu \varepsilon / \mathrm{N}$, respectively.

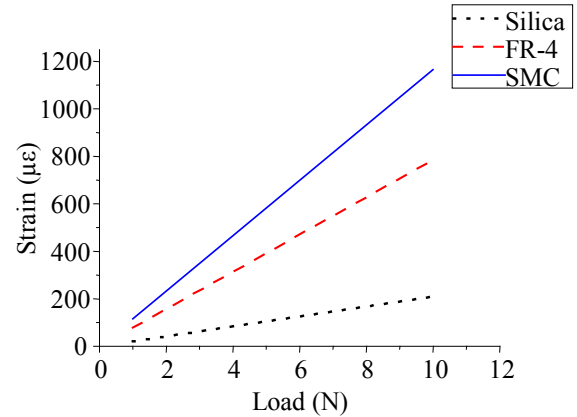

Fig. 4 Strain as a function of the applied load for sensors packaged with three materials.

\section{Experimental results and analysis}

The packaging structure, same as that used in the simulations, was fabricated with three different materials, as shown in Fig. 5. FBGs were fixed on the substrates with ultraviolet glues.

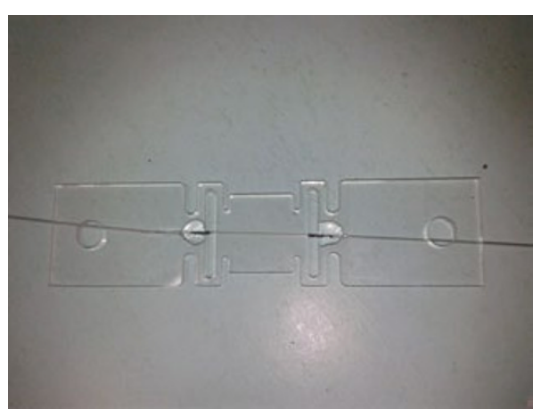

(a) FBG strain sensor packaged with silica

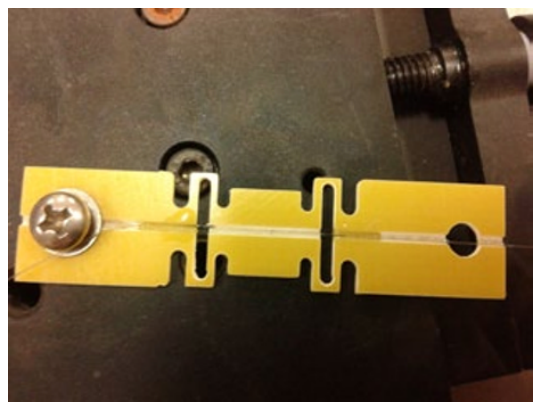

(b) FBG strain sensor packaged with FR-4

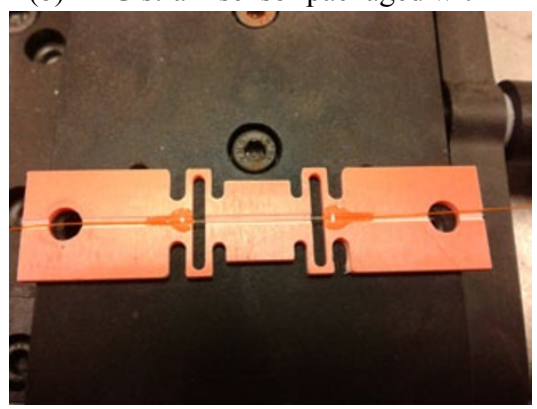

(c) FBG strain sensor packaged with SMC

Fig. 5 FBG strain sensors packaged with three materials. 
One hole was fixed with a screw, and the load was applied on the other hole. The Bragg wavelength was recorded by an optical interrogator while changing the applied load. The Bragg wavelength shift as a function of the load is shown in Fig. 6.

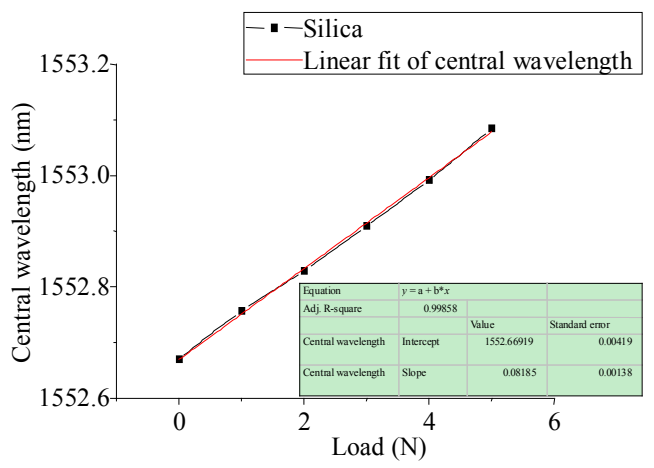

(a) Packaged with silica

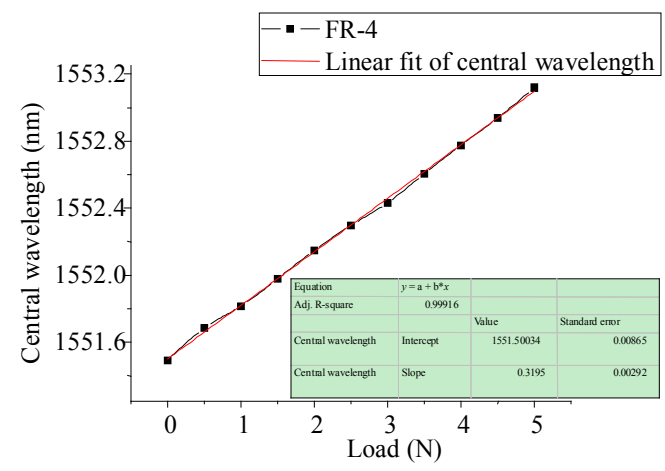

(b) Packaged with FR-4

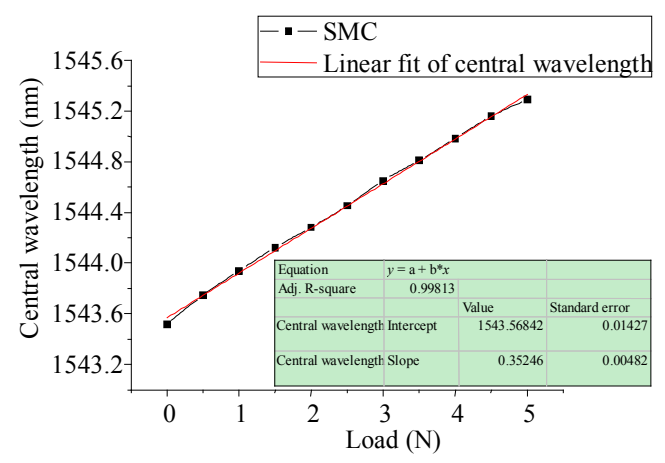

(c) Packaged with SMC

Fig. 6 Bragg wavelength shift as a function of the load.

According to the experimental results, the sensitivities of three sensors packaged with silica, FR-4 and SMC were $81.9 \mathrm{pm} / \mathrm{N}, 319.5 \mathrm{pm} / \mathrm{N}$ and $352.5 \mathrm{pm} / \mathrm{N}$, respectively. The linearities were 0.9986, 0.9992 and 0.9981, respectively. According to the simulated results, the wavelength-strain sensitivities of the FBG sensors with different packaging materials were $3.754 \mathrm{pm} / \mu \varepsilon, 3.02 \mathrm{pm} / \mu \varepsilon$ and $3.03 \mathrm{pm} / \mu \varepsilon$, respectively. The sensitivities were $3.13,2.52,2.52$ times higher than those of pure FBG sensors. As the Young's modulus of the packaging material decreased, the amplification factor of the wavelength-strain sensitivity decreased. However, in the experiments, the silica substrate was easy to be broken due to its brittle texture and poor ductility. Totally speaking, the GFRP is preferred for packaging FBG sensors that used in the marine applications.

\section{Conclusions}

The fiber Bragg grating strain sensors packaged with three corrosion-resistant materials were investigated both numerically and experimentally. The wavelength-strain sensitivities of the three FBG sensors were $3.76 \mathrm{pm} / \mu \varepsilon, 3.02 \mathrm{pm} / \mu \varepsilon$ and $3.03 \mathrm{pm} / \mu \varepsilon$, respectively, and the linearity of each sensor was better than 0.998. In view of the corrosion-resistance of the nonmetallic materials, the fiber Bragg grating strain sensors packaged with GFRP materials may find wide applications in the structural health monitoring for the offshore drilling platform.

Open Access This article is distributed under the terms of the Creative Commons Attribution License which permits any use, distribution, and reproduction in any medium, provided the original author(s) and source are credited

\section{References}

[1] Y. J. Rao, A. B. Lobo Ribeiro, D. A. Jackson, L. Zhang, and I. Bennion, "Simultaneously spatial-, time-, and wavelength-division multiplexed in-fiber Bragg grating sensor network," in Proc. SPIE in Distributed and Multiplexed Fiber Optic Sensors VI, Denver , USA, Aug. 4, vol. 2838, pp. 23-30, 1996.

[2] M. LeBlanc, S. Y. Huang, M. Ohn, R. M. Measures, A. 
Guemes, and A. Othonos, "Distributed strain measurement based on a fiber Bragg grating and its reflection spectrum analysis," Optics Letters, vol. 21, no. 17 , pp. 1405-1407, 1996.

[3] A. S. Rodrigo and A. L. Roberto, "Structural health monitoring of marine composite structural joints using embedded fiber Bragg grating strain sensors," Composite Structures, vol. 89, no. 2, pp. 224-234, 2009.

[4] K. T. Lau, L. M. Zhou, and L. Ye. "Investigation on upgrading and health monitoring the civil concrete structures using FRP and FBG sensor," Advanced Composites Letters, vol. 8, no. 6, pp. 323-332, 1999.
[5] Z. L. Yuan, Y. Gong, Y. Y. Ma, L. Du, Y. Wu, Y. J. Rao, et al., "Structure optimization of fiber Bragg grating strain sensors", Acta Photonica Sinica, vol. 41, no. 11, pp. 1261-1266, 2012.

[6] Y. J. Rao, "In-fibre Bragg grating sensors," Measurement Science and Technology, vol. 8, no. 4, pp. 355-375, 1997.

[7] A. Kerrouche, W. J. O. Boyle, T. Sun, and K. T. V. Grattan, "Design and in-the-field performance evaluation of compact FBG sensor system for structural health monitoring applications," Sensors and Actuators A: Physical, vol. 151, no. 2, pp. 107-112, 2009. 